Graf-Schlicker (Hrsg.)

Insolvenzordnung 



\title{
InsO
}

\section{Kommentar zur Insolvenzordnung}

\author{
5. Auflage
}

Herausgegeben von

Ministerialdirektorin a. D. Marie Luise Graf-Schlicker, Berlin

Bearbeitet von

Alexander Bornemann, Dr. Bettina E. Breitenbücher, Michael Bremen, Dr. Klaus-Peter Busch, Hans Werner Castrup, Ben Dany, Marie Luise Graf-Schlicker, Dr. Matthias Hofmann, Prof. Dr. Michael Huber, Dr. Frank Kebekus, Thomas Kexel, Alexander Kubusch, Marc D. Lienau, Prof. Dr. Anette U. Neußner, Dr. Uwe Paul, Ernst Riedel, Oliver Sabel, Claudia Steh, Rudolf Voß, Dr. Benjamin Webel, Marcus Wehler

\section{RWS}

RWS Verlag Kommunikationsforum GmbH \& Co. KG · Köln 
Die Deutsche Bibliothek verzeichnet diese Publikation in der Deutschen Nationalbibliografie; detaillierte bibliografische Daten sind im Internet über http://dnb.ddb.de abrufbar.

(c) 2020 RWS Verlag Kommunikationsforum GmbH \& Co. KG Postfach 2701 25, 50508 Köln

E-Mail: info@rws-verlag.de, Internet: http://www.rws-verlag.de

Das vorliegende Werk ist in all seinen Teilen urheberrechtlich geschützt. Alle Rechte vorbehalten, insbesondere das Recht der Übersetzung, des Vortrags, der Reproduktion, der Vervielfältigung auf fotomechanischem oder anderen Wegen und der Speicherung in elektronischen Medien.

Satz und Datenverarbeitung: SEUME Publishing Services GmbH, Erfurt Druck und Bindung: CPI books GmbH, Leck 